curriculum makes a specific statement regarding the importance of mental health and specifies a syllabus covering this important area of medical practice

Around $80 \%$ of doctors are expected to have exposure to a community medicine placement, with around $40 \%$ expected to have placement within a specific mental health setting. Though other community placements may provide some exposure to the acute challenges of mental health, this is not guaranteed.

To assist in meeting the FP requirement for training in mental health we developed an online resource for all West Midlands trainees, with lectures and information available that covers all of the core curriculum requirements for the FP. These resources can be accessed at any time of the day, at any point of foundation training, with each module certificated to show evidence of the attainment of foundation competencies ready for students ARCP (Annual Review of Competency Progression).

Result. A programme of evaluation and effectiveness will be undertaken when the new curriculum goes live.

Conclusion. There is an expected expansion in the number of training Foundation doctors within the next 5 years; therefore the demand for this training is expected to increase over time. As the understanding and awareness of the interaction between physical health and mental health continues to develop, we expected the use of this resource grow into the future.

\section{An innovative method of expanding the support for doctors returning to training in psychiatry after a period of extended leave: the Sheffield Mindful Support Programme}

Helen Linnington* and Hamid Alhaj

Sheffield Health and Social Care NHS Foundation Trust ${ }^{*}$ Corresponding author.

doi: 10.1192/bjo.2021.409

Aims. To offer regular continuous professional development opportunities covering both clinical and non-clinical skills to trainees and trainers and enhance their experience and skills to increase their wellbeing and resilience.

Background. There are approximately 50,000 doctors undertaking postgraduate training in England. Of these, 10\% (5000) are taking approved time out of training at any time. A 2017 HEE survey revealed that doctors returning to work reported numerous concerns. Based on these and with the backdrop of the Bawa-Gaba case HEE's Supported return to Training programme (SuppoRTT) was developed.

We at Sheffield Health and Social Care NHS Foundation Trust devised a unique "Mindful SuppoRTT" initiative and were successful in securing funding from HEE. Part of which was the organisation of a conference aimed at various groups of doctors including those who have previously had time out of training, are currently out of training and those considering time out.

The Sheffield Mindful SuppoRTT Programme not only aimed to provide a structured and systematic process for planning and returning from absence, but also focussed on enhancing performance through promoting the wellbeing of participants and supporting them with important clinical and non-clinical skills.

Method. 2-day twice yearly conferences, which covered training on speciality specific as well as non-technical skills were organised. The clinical workshops covered interactive sessions of common and emergency clinical scenarios. A wide range of non-technical skills such as an introduction to mindfulness, tai chi, resilience, team-working and leadership, "Thinking Environment" and meditation were introduced and developed using bespoke training.
Feedback was collected at the end of each conference day. The attendees were asked to use a 5 -point Likert scale ( 5 being the highest) to rate their satisfaction with the day and to highlight which sessions they found most and least useful.

Result. The attendee satisfaction rate was high. The first conference had ratings of $56 \%$ of attendees scoring 5 (excellent) and the remainder scoring 4 (very good). The second conference achieved even higher satisfaction ratings with $94 \%$ of attendees scoring 5 and the remainder scoring 4.

Conclusion. The conference had high attendee satisfaction. The hope is to expand on its success and open it up to delegates from all specialities within HEE South Yorkshire and the Humber. Evaluation of the long-term impact of this programme is also warranted.

\section{An evaluation of the Royal College of Psychiatrists' "Psych Star" scheme}

India Lunn ${ }^{1 \star}$ and Declan Hyland ${ }^{1}$

${ }^{1}$ Medical undergraduate, University of Sheffield and ${ }^{2}$ Clock View Hospital, Liverpool, Mersey Care NHS Foundation Trust ${ }^{\star}$ Corresponding author.

doi: 10.1192/bjo.2021.410

Aims. In 2019, the Royal College of Psychiatrists (RCPsych) launched the "Psych Star" scheme for medical students with an interest in psychiatry. The one-year scheme provides Psych Stars with a matched mentor, free registration at the RCPsych's International Congress, financial support for psychiatry-related activities, journal subscriptions and access to two online learning platforms. This project aimed to evaluate the effectiveness of the scheme in supporting Psych Stars to explore their interest in psychiatry and in promoting psychiatry as a career choice, through use of a survey for both Psych Stars and mentors.

Method. Surveys were sent to all Psych Stars and mentors from the first cohort of the scheme. The mentor and student surveys contained a mixture of ranking, multiple choice, closed-ended and open-ended questions. Questions examined: clarity of the scheme's aims and objectives; benefits of each aspect of the scheme; impact of the scheme on application to Core Training; benefits and barriers to successful mentorship; adequacy of mentor support from the RCPsych and suggestions to improve the scheme.

Result. Six Psych Stars and nine mentors completed the respective surveys. All Psych Stars stated the scheme was enjoyable. Five Psych Stars were more likely to apply for Core Training because of the scheme. All Psych Stars identified the most beneficial aspect of the scheme being the funded place at the RCPsych International Congress. Other aspects highly ranked included: funding to explore areas of psychiatry of interest and the opportunity to be an ambassador for psychiatry. All Psych Stars found the mentorship useful.

Mentors supported Psych Stars by providing career advice, suggesting relevant conferences to attend and assisting Psych Stars make decisions on how to use their allocated funding. Barriers to mentorship that were identified included: geographical separation, limitations related to the COVID-19 pandemic and lack of time. For mentors, areas for improvement included clearer aims and objectives and more support from the RCPsych.

Both mentors and Psych Stars suggested forming a network of Psych Stars and mentors would be useful to share ideas and experiences.

Conclusion. This evaluation shows that the Psych Star scheme successfully supports Psych Stars to explore their interest in 
psychiatry, and promotes psychiatry as a career choice. This survey has been helpful in identifying what aspects of the scheme are particularly attractive, and also, importantly, how the Psych Stars scheme can be improved for future cohorts. The survey will be delivered to all future annual cohorts of Psych Stars and mentors.

\section{Digitalised remote-delivery of AVATr Simulation in Psychiatry: a unique success in COVID-19 pandemic \\ Vimal Mannali $^{1 \star}$, Paul Strickland ${ }^{2}$, Craig laBuscagne ${ }^{1}$ and Joy Clift ${ }^{1}$ \\ ${ }^{1}$ Surrey and Borders Partnership NHS Foundation Trust and \\ ${ }^{2}$ Xenodu Virtual Environments \\ ${ }^{\star}$ Corresponding author.}

\section{doi: 10.1192/bjo.2021.411}

Aims. Surrey and Borders NHS Foundation Trust's AVATr (Augmented Virtual-reality Avatar in Training) is a unique ground-breaking Virtual Patient Simulation System, which uses the Xenodu platform to train learners in essential clinical and complex communication skills. Over 30 patient scenarios have been developed after identifying learner-specific development needs, including exploration of overt psychosis, assessment of capacity, sharing bad news, and neglect in care home residents. Actors are filmed responding to several domains of clinical questions, further categorised into three narrative-modes of being 'Engaged, Neutral or Disengaged', to build a bank of scenarios. During the session, the trainee is projected on to a large screen, using a camera and video special effects, which results in a life-like interaction with the Virtual Patient. Trainees can view themselves interacting with the Virtual Patient in real-time, from a unique 'out-of-body' perspective, immersed in a custom-designed interactive virtual environment. The simulation facilitator engages with the learner and determines the appropriate choices of responses for the Virtual Patient and if needed, can prompt with explorative cues to continue the narrative-linked conversation. AVATr model pioneered in United Kingdom the use of an innovative 'self-observational approach' in Psychiatry training. This is different to a first-person perspective used in virtual or augmented-reality systems in several clinical specialties. The use of Facilitated-Debrief and Peer-Debrief in sessions, render another layer to the simulation experience.

Method. During the COVID-19 pandemic, we evolved the AVATr model to remote or hybrid sessions, where simulations were digitally enhanced, and have been run through Microsoft Teams. The simulation facilitator is connected to a multi-user video call, enabling the Virtual Patient to be projected as an attendee using Microsoft Teams.

Result. The hybrid model of AVATr has received tremendous feedback, as it now simulates video-consultations that a vast majority of Psychiatry trainees, especially community-based, undertake due to COVID-19 restrictions. The format of AVATr simulation sessions has remained unchanged, and the remote delivery has been particularly successful as it allows trainees to log in from different remote locations to come together for an interactive training session, without any physical restrictions.

Conclusion. Since 2015, our simulation platform has been utilised for Post-Graduate Medical Education, to enhance essential professional skills and stimulate professional growth. Currently the hybrid model of AVATr is being expanded to Nursing, Psychology and Allied Health Professional (AHP) clinical training streams, along with Undergraduate Medical Education, to address identified gaps in face-to-face training amidst COVID-19 pandemic.
A cross-sectional study exploring attitudes of first year medical students towards psychiatry and factors they identified that would help stimulate their interest

Isabel Mark* and Victoria Fernandez Garcia De Las Heras

South West London and St George's Mental Health Trust, St George's University of London

${ }^{\star}$ Corresponding author.

doi: 10.1192/bjo.2021.412

Aims. Psychiatry has had long standing recruitment difficulties. Many efforts have been made to explore strategies that encourage interest in the specialty, with early university experience being an important factor in ultimate career choice. The Royal College of Psychiatrists 'Choose Psychiatry' guidance for medical schools outlines four key areas of focus: teaching excellence, placement quality, leadership and enrichment activities, with other research reporting similar conclusions. The aim of this study was to assess attitudes towards psychiatry amongst first year medical students, examine what input they would welcome from psychiatrists at this stage of their career and consider if their wishes are in keeping with the 'Choose Psychiatry' guidance.

Method. All first-year medical students at St George's University of London were approached in October 2019 and offered the opportunity for early psychiatry exposure. 60 students were recruited. Data were collected in November 2019 via an online questionnaire comprising of baseline demographics, the 30-item Attitudes Towards Psychiatry questionnaire (ATP-30) and a freetext question asking what students would like from psychiatry at this stage of their education. Quantitative data were analysed using Excel, whilst qualitative data were analysed thematically.

Result. The mean ATP-30 score was 113.83 (SD 12.57, range 70-135). Gender, ethnicity and religious background were not associated with a change in ATP score. Undergraduates' attitudes were more positive than those of postgraduates (independent $\mathrm{t}$-test revealed a p-value of 0.087 ). Seven themes were identified outlining what students wanted from psychiatry, the most prominent being: (a) learning about the lifestyle of a psychiatrist and finding a role model, (b) exploring the patient perspective, (c) exploring the interaction between psychiatry and specialities and (d) having an opportunity to develop communication skills.

Conclusion. The findings demonstrate higher ATP-30 results than previous literature has reported, potentially due to mental health awareness campaigns in recent years. As undergraduates were found to have a higher mean score, targeting them for additional psychiatry contact may be beneficial. Themes identified by students in this study support 'Choose Psychiatry' guidance, whilst also highlighting the potential for combining some psychiatry with other specialties in the curriculum. Integration with communication skills teaching might help engage those not initially psychiatry-inclined. Further study will be required to establish whether implementing the suggested strategies can lead to improvement in student ATP-30 scores and ultimately increased recruitment rates.

Increasing staff confidence about domestic abuse identification, disclosure and safeguarding in a community mental health team

Beth McCausland ${ }^{1 *}$, Nicola Minicozzi ${ }^{1}$, Siobhan O'Halloran ${ }^{1}$, Avril Ward ${ }^{2}$ and Kerry Elliott ${ }^{3}$

${ }^{1}$ Southern Health NHS Foundation Trust, University of Southampton; ${ }^{2}$ Yellow Door, Pathfinder and ${ }^{3}$ Aurora New Dawn Ltd, Pathfinder

${ }^{\star}$ Corresponding author.

doi: 10.1192/bjo.2021.413 